\title{
Prognostic significance of red cell distribution width in bladder cancer
}

\author{
Wenchao Ma ${ }^{1,2 \#}$, Shiyu $\mathrm{Mao}^{2 \#}$, Meiyu Bao ${ }^{3 \#}$, Yuan $\mathrm{Wu}^{1}$, Yadong Guo ${ }^{2}$, Ji Liu ${ }^{2}$, Ruiliang Wang ${ }^{2}, \mathrm{Cheng}^{2}$, \\ Junfeng Zhang ${ }^{2}$, Wentao Zhang ${ }^{2}$, Xudong Yao ${ }^{1,2}$ \\ ${ }^{1}$ Shanghai Clinical College, Anhui Medical University, Hefei 230032, China; ${ }^{2}$ Department of Urology, Shanghai Tenth People's Hospital, Tongji \\ University, Shanghai 200072, China; ${ }^{3}$ Department of Central Laboratory, Shanghai Tenth People's Hospital, Tongji University, Shanghai 200072, \\ China \\ Contributions: (I) Conception and design: W Ma; Wo Zhang; (II) Administrative support: X Yao; J Zhang; (III) Provision of study materials or \\ patients: S Mao; (IV) Collection and assembly of data: J Liu; Y Wu; M Bao; C Li; (V) Data analysis and interpretation: Y Guo; R Wang; W Ma; (VI) \\ Manuscript writing: All authors; (VII) Final approval of manuscript: All authors. \\ \#These authors contributed equally to this work. \\ Correspondence to: Prof. Xudong Yao; Prof. Wentao Zhang. Department of Urology, Shanghai Tenth People's Hospital, Tongji University, Shanghai \\ 200072, China. Email: yaoxudong1967@163.com; zhangwentao98@163.com.
}

Background: Bladder cancer is one of the most common cancers worldwide. It ranks ninth among all cancers and fourth among cancers in male patients. Recent studies reported that red blood cell (RBC) distribution width (RDW) was a potential predictor in some cancers. This study explored the significance of RDW in patients with bladder cancer after radical cystectomy.

Methods: This study involved 169 patients who underwent radical cystectomy between March 2009 and October 2018. The overall survival (OS), cancer-specific survival (CSS), and disease-free survival (DFS) were analyzed using the Kaplan-Meier method. Univariate and multivariate Cox analyses were used to evaluate the prognostic significance of RDW.

Results: The patients with bladder cancer were divided into two subgroups according to the RDW value (0.1395). A high RDW value significantly correlated with higher mortality, a decrease in hemoglobin, an increase in $\mathrm{C}$-reactive protein, a decrease in $\mathrm{RBC}$ count, and $\mathrm{T}$ stage $(\mathrm{P}<0.05)$. Statistically significant differences in OS, CSS, and DFS were found between high- and low-RDW groups. Hence, a high RDW value was presumed to be a risk factor for poor prognosis in patients with bladder cancer after radical cystectomy $(\mathrm{P}<0.001)$.

Conclusions: Patients with a high RDW value had a poor prognosis. Therefore, RDW is a reliable predictor for the prognosis of patients with bladder cancer who underwent radical cystectomy.

Keywords: Bladder cancer; prognosis; red blood cell distribution width (RDW); radical cystectomy; systemic inflammation

Submitted Nov 02, 2019. Accepted for publication Feb 14, 2020.

doi: 10.21037/tau.2020.03.08

View this article at: http://dx.doi.org/10.21037/tau.2020.03.08

\section{Introduction}

Bladder cancer is one of the most common cancers worldwide. It ranks ninth among all cancers and fourth among cancers in male patients $(1,2)$. Patients are often treated for painless hematuria, which is the most common symptom of bladder cancer (3). About three fourths patients have nonmuscle-invasive bladder cancer, and the rest have muscle-invasive bladder cancer (4). A predictor to evaluate bladder cancer is urgently required due to poor prognosis and high recurrence. 
The prognosis of patients with cancer is not only determined by cancer itself but also associated with many other factors, such as nutritional and physical statuses, mentality, age, and so on $(5,6)$. Meanwhile, systemic inflammation related to cancer is also an important prognostic factor in different cancers (7). The inflammatory reaction changes with the development of cancer (8). Hence, the inflammation index can reflect a patient's status and may be used as a predictor to forecast patient's prognosis (9). Several studies indicated a close correlation of red blood cell (RBC) distribution width (RDW) with systemic inflammation, reflecting the inflammation status due to cancer. RDW is a parameter that reflects the volume heterogeneity of RBCs and it can be calculated from the standard deviation (SD) of erythrocyte volumes for the mean corpuscular volume (MCV) $(10,11)$. It is always expressed by the coefficient of variation of the erythrocyte volume in the blood routine test sheet. Inflammation, nutritional disorders and changes in erythropoiesis may alter RDW, which usually affects bone marrow and erythropoietin, a hormone that regulates the production, maturation and survival of RBCs (12). RDW is conventionally used to evaluate hematological diseases, especially anemia (13). In recent years, RDW has been reported as a biomarker of carcinoma (14-17). However, the correlation of RDW with bladder cancer is still not clear. Therefore, this study was conducted to explore whether RDW could serve as a predictor for the prognosis of bladder cancer by analyzing the data of patients who underwent radical cystectomy.

\section{Methods}

\section{Study design and patients}

A retrospective analysis was performed after preliminarily processing the raw data. It involved 240 patients who underwent radical cystectomy between March 2009 and October 2018 at the Shanghai Tenth People's Hospital (Shanghai, China). The inclusion criteria were as follows: (I) patients diagnosed with primary transitional cell carcinoma; (II) RDW value detected from whole blood samples; (III) RDW value detected within 1 week before the surgery; (IV) all patients hospitalized and the follow-up data completed; (V) none of the patients having systemic disease; (VI) all patients undergoing radical cystectomy within 3 weeks from admission; and (VII) no death within 30 days after the surgery; (VIII) patients undergoing neoadjuvant chemotherapy were excluded in our study. After filtering,
169 patients were ultimately included in the study. The study was approved by the Ethics Committee of the Tenth People's Hospital of Shanghai (SHSY-IEC-4.1/19-120/01), and all patients signed a written informed consent form. This study was also in line with the Helsinki declaration.

\section{Data collection}

Two experienced doctors collected, recorded, and analyzed data, including demographic parameters, tumor-related parameters, and laboratory tests. All blood samples were collected before the patient underwent a radical cystectomy. The overall survival (OS) is the time from the accomplishment of the surgery to the date of the death irrespective of causes. The cancer-specific survival (CSS) is the time from the completion of the surgery to the death of patients due to bladder cancer. Disease-free survival (DFS) is the time when a patient survives without any signs or symptoms of cancer after the surgery.

\section{Statistical analysis}

IBM SPSS Statistics 22.0 software was used for statistical analysis. The Kolmogorov-Smirnov test was used to evaluate normality. Patients were divided into two groups according to RDW values to find out the relationship between RDW and prognosis in different subgroups. The correlation between the RDW value and clinicopathological parameters was evaluated using the chi-square test, Student's $t$-test, and Mann-Whitney $U$ test. Univariate and multivariate Cox regression analyses were used to analyze the relationship between RDW and OS, DFS, and CSS of patients after radical cystectomy. Kaplan-Meier curves tested with the log-rank test were used to evaluate the patient survival status. The risk ratio estimated according to the Cox analysis was reported as a hazard ratio, with a corresponding $95 \%$ confidence interval. A $\mathrm{P}$ value less than 0.05 was considered to be statistically significant.

\section{Results}

\section{Demographic characteristics of patients}

A total of 169 patients (145 male and 24 female) with bladder cancer were eligible for the study. The average age of the participants was 66.65 years (range, 32-87 years). The average follow-up time was 32.32 months (range, 2108 months). Further, 51 patients died during the follow-up 
period. Among the 169 patients, $36.1 \%$ had a solitary tumor and $63.9 \%$ had multiple bladder tumors. The baseline and clinicopathological characteristics of the patients are shown in Table 1.

\section{Optimal cutoff value of RDW and clinicopathological features of patients}

The patients were divided into two subgroups according to the RDW value. The $\mathrm{X}$-tile software (Developed by Yale University) was used to determine the cutoff value. The value in the high-RDW subgroup was more than 0.1395 , and the value in the low-RDW subgroup was less than 0.1395 . The increase in the RDW value significantly correlated with higher mortality, a decrease in hemoglobin, an increase in C-reactive protein (CRP), a decrease in the $\mathrm{RBC}$ count, and $\mathrm{T}$ stage $(\mathrm{P}<0.05)$. It was not related to sex, age, tumor size, $\mathrm{N}$ stage, diabetes, hypertension, cardiovascular and cerebrovascular diseases, and single/ multiple, primary, or recurrent tumors $(\mathrm{P}>0.05)$. More details are displayed in Table 1.

\section{Univariate and multivariate analyses of OS, CSS, and DFS based on RDW}

The univariate Cox proportional model was used to analyze factors affecting OS, CSS, and DFS in patients with bladder cancer. The results showed that age, $\mathrm{T}$ stage, $\mathrm{N}$ stage, RDW, erythrocyte count, CRP, and hemoglobin were significantly related to OS; T stage, $\mathrm{N}$ stage, RDW, RBC count, CRP, and hemoglobin were significantly related to CSS; and T stage, N stage, RDW, RBC count, CRP, and hemoglobin were significantly related to DFS. The multivariate Cox analysis showed that age, $\mathrm{N}$ stage, RDW, and CRP were independent prognostic factors for OS in patients after radical cystectomy (Table 2). N stage, RDW, and CRP were independent prognostic factors for CSS and DFS in patients with bladder cancer (Tables 3,4).

Kaplan-Meier curves showed statistically significant differences in OS, CSS, and DFS between high- and lowRDW groups (Figure 1). A high RDW value might be a risk factor for poor prognosis in patients after radical cystectomy $(\mathrm{P}<0.001)$. Therefore, RDW can be used as a clinical marker to guide the work of clinicians.

\section{Discussion}

The present study found that patients with a high RDW value had a poor prognosis, which correlated with a high $\mathrm{T}$ stage and a low RBC count and hemoglobin. The multivariate analysis showed that RDW, CRP, and N stage statistically significantly correlated with OS, CSS, and DFS. RDW, CRP, and $\mathrm{N}$ stage might be independent risk factors for OS, CSS, and DFS in patients undergoing radical cystectomy.

Recently, some studies explored whether RDW could predict the prognosis of patients with cancers. Goyal et al. reported that RDW might serve as a predictor to predict the prognosis of patients with hepatocellular carcinoma (18). The advantage of their investigation was that they summarized many studies. Cheng et al. demonstrated that a high RDW value always meant poor prognosis in patients with upper tract urothelial carcinoma (19). The limitation of this study was that the diagnosis of patients is now almost ten years away. Wang et al. reported that RDW was a potential prognostic biomarker for esophageal squamous cell carcinoma, which was identified by a nomogram (20). Their study included more than 2,000 patients, and a nomogram was constructed to predict the prognosis of patients.

Several studies indicated a close correlation between RDW and systemic inflammation, which reflected the inflammation status of the body (21). Systemic inflammation has been considered to be closely related to the occurrence and development of tumors in recent years. Some inflammatory indicators have been reported to serve as a potential biomarker of prognosis and correlate with some solid tumors $(13,22,23)$. Cancer can induce chronic inflammation and cause the release of tumor necrosis factoralpha, interleukin 6 (IL-6), and other cytokines $(24,25)$. For example, activated IL-6 can prompt epithelial-mesenchymal transition in bladder cancer (26). IL-10 can promote the expression of PD-L1 of macrophages in the tumor microenvironment to induce T-cell immunosuppression in bladder cancer (27). Another study has also reported that IL-10 can inhibit macrophages to protect bladder cancer cells (28). IL-17 can accelerate tumor growth through the IL-6/STAT3 signaling pathway (29). Excluding these aspects, pretreatment serum IL levels can predict the progression of some cancers, such as colorectal cancer (30).

This study also found statistically significant differences in CRP, RBC count, hemoglobin, and T stage between the two subgroups. CRP is an acute-phase substance with elevated levels in systemic infection and malignant tumors. Previous studies reported that CRP might predict the prognosis of patients with urinary tract urothelial 
Table 1 Relationships between RDW and clinicopathological characteristics of 169 patients with bladder cancer

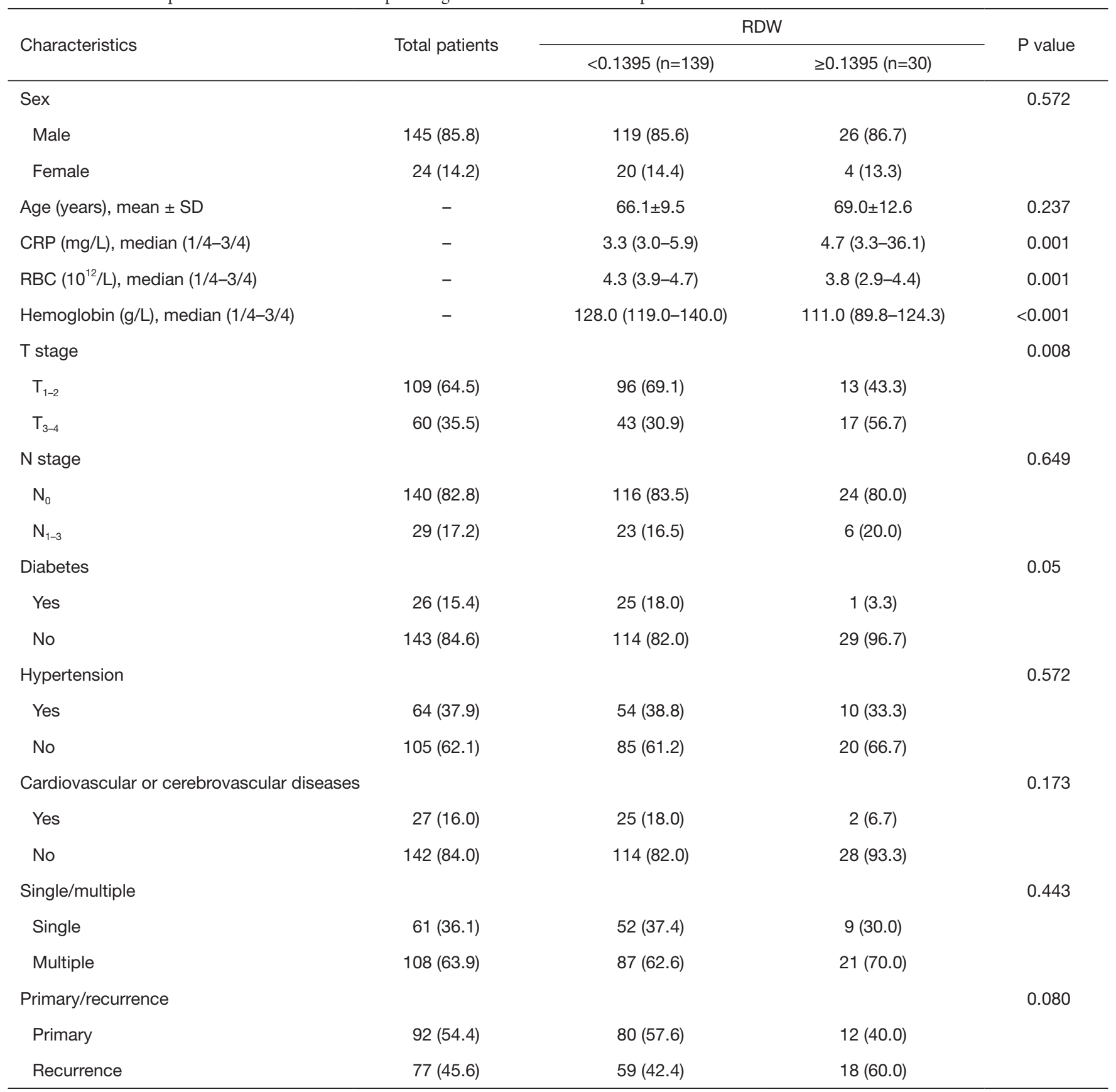

Single: single bladder cancer mass; multiple: two or more bladder cancer masses; primary: primary bladder cancer; recurrence: recurrent bladder cancer. RDW, red blood cell distribution width; SD, standard deviation; CRP, C-reactive protein; RBC, red blood cell.

carcinoma (31-33).

However, the present study had some limitations. First, it was a retrospective study of a single institution design, and the sample size was small. Second, the follow-up time was quite short. Finally, it did not explore the biological interaction between systemic inflammation and cancer. Hence, more multicenter and large-sample prospective studies are required in the future to validate the findings.

In conclusion, RDW has significance as a predictor in patients with bladder cancer who underwent radical 
Table 2 Prognostic factors for overall survival in 169 patients with bladder cancer

\begin{tabular}{|c|c|c|c|c|c|c|}
\hline Variables & $\begin{array}{l}\text { Patients } \\
(n=169)\end{array}$ & $\begin{array}{l}\text { Category or } \\
\text { characteristics }\end{array}$ & \multicolumn{2}{|l|}{ Univariate } & \multicolumn{2}{|c|}{ Multivariate } \\
\hline Sex & $24 / 145$ & Female/male & $0.902(0.408-1.995)$ & 0.799 & - & - \\
\hline Age, years & $90 / 79$ & $>65 / \leq 65$ & 2.165 (1.238-3.789) & 0.007 & $1.029(1.001-1.058)$ & 0.044 \\
\hline T stage & $109 / 60$ & $\mathrm{~T}_{1-2} / \mathrm{T}_{3-4}$ & 2.668 (1.574-4.522) & $<0.001$ & $1.399(0.734-2.665)$ & 0.308 \\
\hline Hypertension & $64 / 105$ & Yes/no & $1.035(0.606-1.768)$ & 0.9 & - & - \\
\hline Diabetes & $26 / 143$ & Yes/no & $1.284(0.661-2.495)$ & 0.46 & - & - \\
\hline $\begin{array}{l}\text { Cardiovascular or } \\
\text { cerebrovascular diseases }\end{array}$ & $27 / 142$ & Yes/no & $0.837(0.409-1.713)$ & 0.627 & - & - \\
\hline Hemoglobin & $36 / 133$ & $\geq 110 /<110$ & $0.368(0.213-0.639)$ & $<0.001$ & $0.998(0.985-1.012)$ & 0.796 \\
\hline Primary/recurrence & $92 / 77$ & Primary/recurrence & $0.973(0.577-1.643)$ & 0.919 & - & - \\
\hline Single/multiple & $61 / 108$ & Single/multiple & $0.598(0.352-1.016)$ & 0.057 & - & - \\
\hline
\end{tabular}

Single: single bladder cancer mass; multiple: two or more bladder cancer masses; primary: primary bladder cancer; recurrence: recurrent bladder cancer. HR, hazard ratio; Cl, confidence interval; RDW, red blood cell distribution width; RBC, red blood cell; CRP, C-reactive protein.

Table 3 Prognostic factors for cancer-specific survival in 169 patients with bladder cancer

\begin{tabular}{|c|c|c|c|c|c|c|}
\hline Variables & $\begin{array}{l}\text { Patients } \\
(n=169)\end{array}$ & $\begin{array}{c}\text { Category or } \\
\text { characteristics }\end{array}$ & \multicolumn{2}{|l|}{ CSS } & \multicolumn{2}{|l|}{ cSs } \\
\hline Sex & $24 / 145$ & Female/male & $0.656(0.199-2.159)$ & 0.488 & - & - \\
\hline Age, years & $90 / 79$ & $>65 / \leq 65$ & $1.942(0.935-4.033)$ & 0.075 & - & - \\
\hline T stage & $109 / 60$ & $\mathrm{~T}_{1-2} / \mathrm{T}_{3-4}$ & $2.020(1.002-4.071)$ & 0.049 & - & - \\
\hline Hypertension & $64 / 105$ & Yes/no & $0.558(0.250-1.245)$ & 0.154 & - & - \\
\hline Diabetes & $26 / 143$ & Yes/no & $1.568(0.677-3.632)$ & 0.293 & - & - \\
\hline $\begin{array}{l}\text { Cardiovascular or } \\
\text { cerebrovascular diseases }\end{array}$ & $27 / 142$ & Yes/no & $0.789(0.303-2.058)$ & 0.629 & - & - \\
\hline Hemoglobin & $36 / 133$ & $\geq 110 /<110$ & $0.429(0.202-0.911)$ & 0.028 & $1.781(0.531-5.965)$ & 0.350 \\
\hline Primary/recurrence & $92 / 77$ & Primary/recurrence & $1.048(0.522-2.106)$ & 0.894 & - & - \\
\hline Single/multiple & $61 / 108$ & Single/multiple & $0.694(0.338-1.422)$ & 0.318 & - & - \\
\hline
\end{tabular}

Single: single bladder cancer mass; multiple: two or more bladder cancer masses; primary: primary bladder cancer; recurrence: recurrent bladder cancer. CSS, cancer-specific survival; HR, hazard ratio; $\mathrm{Cl}$, confidence interval; RDW, red blood cell distribution width; RBC, red blood cell; CRP, C-reactive protein. 
Table 4 Prognostic factors for disease-free survival in 169 patients with bladder cancer

\begin{tabular}{|c|c|c|c|c|c|c|}
\hline Variables & $\begin{array}{l}\text { Patients } \\
(n=169)\end{array}$ & $\begin{array}{l}\text { Category or } \\
\text { characteristics }\end{array}$ & \multicolumn{2}{|l|}{ DFS } & \multicolumn{2}{|l|}{ DFS } \\
\hline Sex & $24 / 145$ & Female/male & $0.870(0.431-1.754)$ & 0.696 & - & - \\
\hline Age & $90 / 79$ & $>65 / \leq 65$ & $1.448(0.897-2.337)$ & 0.129 & - & - \\
\hline T stage & $109 / 60$ & $\mathrm{~T}_{1-2} / \mathrm{T}_{3-4}$ & 2.677 (1.662-4.310) & $<0.001$ & $1.466(0.828-2.597)$ & 0.190 \\
\hline Hypertension & $64 / 105$ & Yes/no & $0.926(0.570-1.505)$ & 0.756 & - & - \\
\hline Diabetes & $26 / 143$ & Yes/no & $1.424(0.788-2.571)$ & 0.242 & - & - \\
\hline $\begin{array}{l}\text { Cardiovascular or } \\
\text { cerebrovascular diseases }\end{array}$ & $27 / 142$ & Yes/no & $0.844(0.443-1.610)$ & 0.607 & - & - \\
\hline CRP & $54 / 115$ & $>5 / \leq 5$ & 2.917 (1.816-4.685) & $<0.001$ & $2.493(1.438-4.321)$ & 0.001 \\
\hline Hemoglobin & $36 / 133$ & $\geq 110 /<110$ & $0.535(0.318-0.901)$ & 0.019 & $1.437(0.618-3.346)$ & 0.400 \\
\hline Primary/recurrence & $92 / 77$ & Primary/recurrence & $1.187(0.742-1.899)$ & 0.474 & - & - \\
\hline Single/multiple & $61 / 108$ & Single/multiple & $0.659(0.408-1.066)$ & 0.089 & - & - \\
\hline
\end{tabular}

Single: single bladder cancer mass; multiple: two or more bladder cancer masses; primary: primary bladder cancer; recurrence: recurrent bladder cancer. DFS, disease-free survival; HR, hazard ratio; Cl, confidence interval; RDW, red blood cell distribution width; RBC, red blood cell; CRP, C-reactive protein.

A

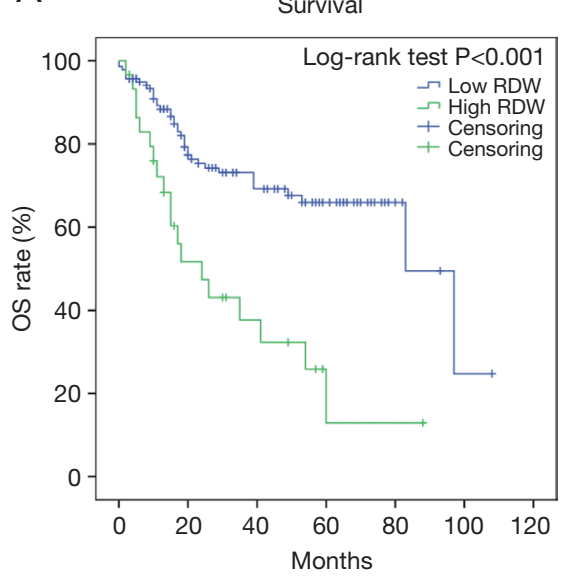

B

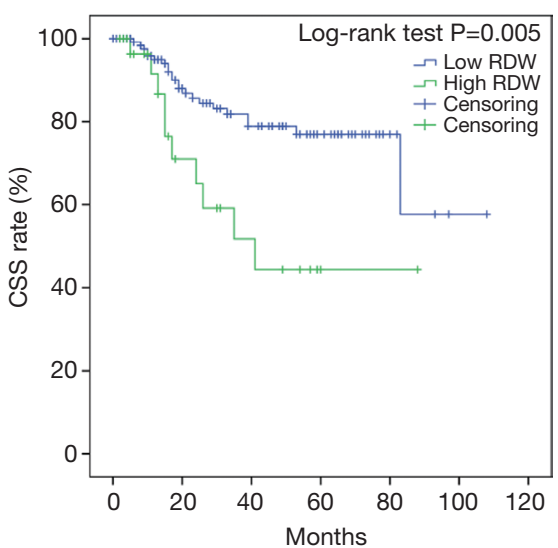

C

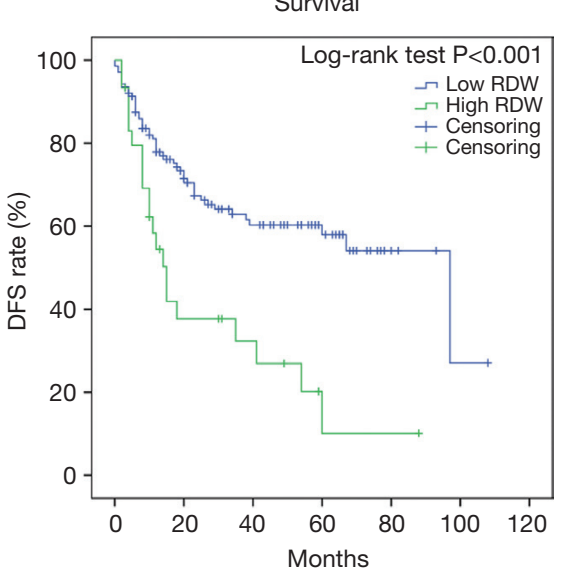

Figure 1 Kaplan-Meier curves of survival based on red blood cell distribution width (RDW) value of 169 patients with bladder cancer. (A) Overall survival (OS); (B) cancer-specific survival (CSS); (C) disease-free survival (DFS). 
cystectomy. However, its specific mechanism of action needs to be explored.

\section{Acknowledgments}

Funding: None.

\section{Footnote}

Conflicts of Interest: All authors have completed the ICMJE uniform disclosure form (available at http://dx.doi. org/10.21037/tau.2020.03.08). The authors have no conflicts of interest to declare.

Ethical Statement: The authors are accountable for all aspects of the work in ensuring that questions related to the accuracy or integrity of any part of the work are appropriately investigated and resolved. The study was approved by the Ethics Committee of the Tenth People's Hospital of Shanghai (SHSY-IEC-4.1/19-120/01), and all patients signed a written informed consent form.

Open Access Statement: This is an Open Access article distributed in accordance with the Creative Commons Attribution-NonCommercial-NoDerivs 4.0 International License (CC BY-NC-ND 4.0), which permits the noncommercial replication and distribution of the article with the strict proviso that no changes or edits are made and the original work is properly cited (including links to both the formal publication through the relevant DOI and the license). See: https://creativecommons.org/licenses/by-ncnd/4.0/.

\section{References}

1. Antoni S, Ferlay J, Soerjomataram I, et al. Bladder Cancer Incidence and Mortality: A Global Overview and Recent Trends. Eur Urol 2017;71:96-108.

2. Siegel RL, Miller KD, Jemal A. Cancer Statistics, 2017. CA Cancer J Clin 2017;67:7-30.

3. Alfred Witjes J, Lebret T, Comperat EM, et al. Updated 2016 EAU Guidelines on Muscle-invasive and Metastatic Bladder Cancer. Eur Urol 2017;71:462-75.

4. Zhang W, Mao S, Shi D, et al. MicroRNA-153 Decreases Tryptophan Catabolism and Inhibits Angiogenesis in Bladder Cancer by Targeting Indoleamine 2,3-Dioxygenase 1. Front Oncol 2019;9:619.

5. Thanikachalam K, Khan G. Colorectal Cancer and
Nutrition. Nutrients 2019. doi: 10.3390/nu11010164.

6. Hoffe S, Balducci L. Cancer and age: general considerations. Clin Geriatr Med 2012;28:1-18.

7. Hirahara N, Tajima Y, Fujii Y, et al. Prognostic significance of red cell distribution width in esophageal squamous cell carcinoma. J Surg Res 2018;230:53-60.

8. Zhong JH, Huang DH, Chen ZY. Prognostic role of systemic immune-inflammation index in solid tumors: a systematic review and meta-analysis. Oncotarget 2017;8:75381-8.

9. Li X, Ma X, Tang L, et al. Prognostic value of neutrophilto-lymphocyte ratio in urothelial carcinoma of the upper urinary tract and bladder: a systematic review and metaanalysis. Oncotarget 2016;8:62681-92.

10. Yang D, Quan W, Wu J, et al. The value of red blood cell distribution width in diagnosis of patients with colorectal cancer. Clin Chim Acta 2018;479:98-102.

11. Danese E, Lippi G, Montagnana M. Red blood cell distribution width and cardiovascular diseases. J Thorac Dis 2015;7:E402-11.

12. Parizadeh SM, Jafarzadeh-Esfehani R, Bahreyni A, et al. The diagnostic and prognostic value of red cell distribution width in cardiovascular disease; current status and prospective. Biofactors 2019;45:507-16.

13. Hirahara N, Tajima Y, Fujii Y, et al. Comprehensive Analysis of Red Blood Cell Distribution Width as a Preoperative Prognostic Predictor in Gastric Cancer. Anticancer Res 2019;39:3121-30.

14. Hsueh CY, Lau HC, Li S, et al. Pretreatment Level of Red Cell Distribution Width as a Prognostic Indicator for Survival in a Large Cohort Study of Male Laryngeal Squamous Carcinoma. Front Oncol 2019;9:271.

15. Ge W, Xie J, Chang L. Elevated red blood cell distribution width predicts poor prognosis in patients with oral squamous cell carcinoma. Cancer Manag Res 2018;10:3611-8.

16. Bozkurt G, Korkut AY, Soytas P, et al. The role of red cell distribution width in the locoregional recurrence of laryngeal cancer. Braz J Otorhinolaryngol 2019;85:357-64.

17. Seretis C, Seretis F, Lagoudianakis E, et al. Is red cell distribution width a novel biomarker of breast cancer activity? Data from a pilot study. J Clin Med Res 2013;5:121-6.

18. Goyal H, Hu ZD. Prognostic value of red blood cell distribution width in hepatocellular carcinoma. Ann Transl Med 2017;5:271.

19. Cheng YC, Huang CN, Wu WJ, et al. The Prognostic Significance of Inflammation-Associated Blood Cell 
Markers in Patients with Upper Tract Urothelial

Carcinoma. Ann Surg Oncol 2016;23:343-51.

20. Wang Y, He SS, Cai XY, et al. The Novel Prognostic Score Combining Red Blood Cell Distribution Width and Body Mass Index (COR-BMI) Has Prognostic Impact for Survival Outcomes in Nasopharyngeal Carcinoma. J Cancer 2018;9:2295-301.

21. Lippi G, Targher G, Montagnana M, et al. Relation between red blood cell distribution width and inflammatory biomarkers in a large cohort of unselected outpatients. Arch Pathol Lab Med 2009;133:628-32.

22. Xie QK, Chen P, Hu WM, et al. The systemic immuneinflammation index is an independent predictor of survival for metastatic colorectal cancer and its association with the lymphocytic response to the tumor. J Transl Med 2018;16:273.

23. Zhang H, Shang X, Ren $P$, et al. The predictive value of a preoperative systemic immune-inflammation index and prognostic nutritional index in patients with esophageal squamous cell carcinoma. J Cell Physiol 2019;234:1794-802.

24. Colotta F, Allavena P, Sica A, et al. Cancer-related inflammation, the seventh hallmark of cancer: links to genetic instability. Carcinogenesis 2009;30:1073-81.

25. Pepys MB, Hirschfield GM. C-reactive protein: a critical update. J Clin Invest 2003;111:1805-12.

26. Zhang $W$, Zhang J, Zhang Z, et al. Overexpression of Indoleamine 2,3-Dioxygenase 1 Promotes EpithelialMesenchymal Transition by Activation of the IL-6/

Cite this article as: $\mathrm{Ma} \mathrm{W}, \mathrm{Mao} \mathrm{S}$, Bao $\mathrm{M}$, Wu Y, Guo Y, Liu J, Wang R, Li C, Zhang J, Zhang W, Yao X. Prognostic significance of red cell distribution width in bladder cancer. Transl Androl Urol 2020;9(2):295-302. doi: 10.21037/ tau.2020.03.08
STAT3/PD-L1 Pathway in Bladder Cancer. Transl Oncol 2019;12:485-92.

27. Wang X, Ni S, Chen Q, et al. Bladder cancer cells induce immunosuppression of $\mathrm{T}$ cells by supporting PD-L1 expression in tumour macrophages partially through interleukin 10. Cell Biol Int 2017;41:177-86.

28. Luo Y, Han R, Evanoff DP, et al. Interleukin-10 inhibits Mycobacterium bovis bacillus Calmette-Guerin (BCG)induced macrophage cytotoxicity against bladder cancer cells. Clin Exp Immunol 2010;160:359-68.

29. Wang L, Yi T, Kortylewski M, et al. IL-17 can promote tumor growth through an IL-6-Stat3 signaling pathway. J Exp Med 2009;206:1457-64.

30. Chang PH, Pan YP, Fan CW, et al. Pretreatment serum interleukin-1beta, interleukin-6, and tumor necrosis factor-alpha levels predict the progression of colorectal cancer. Cancer Med 2016;5:426-33.

31. Stein B, Schrader AJ, Wegener G, et al. Preoperative serum C- reactive protein: a prognostic marker in patients with upper urinary tract urothelial carcinoma. BMC Cancer 2013;13:101.

32. Huang J, Baum Y, Alemozaffar M, et al. C-reactive protein in urologic cancers. Mol Aspects Med 2015;45:28-36.

33. Guo Y, Cai K, Mao S, et al. Preoperative C-reactive protein/albumin ratio is a significant predictor of survival in bladder cancer patients after radical cystectomy: a retrospective study. Cancer Manag Res 2018;10:4789-804. 